\title{
Delexicalizing Features Analysis of Mandarin Chinese Light Verbs
}

\author{
Lin XUE \\ University of Science and Technology Beijing, China
}

\begin{abstract}
Delexicalization is the meaning tendency and structure feature of light verbs. It is the result of collocation usages meaning sharing. Based on corpus and exemplified by "da" and "chi", this paper notes the delexicalizing features of Chinese light verbs. This article develops the lexicographic approach regarding conventionality of light verb construction and attempts to apply it to foreign language acquisition.
\end{abstract}

KEYWORD: Light verb; Mandarin Chinese; Delexicalization

\section{INTRODUCTION}

Light verbs function both as full lexical units and as semi-lexical ones. Dictionary entries dedicated to them are always long and far from exhaustive. Light verbs (LVs), a term launched by generative grammarians and used by many followers (Jespersen, 1965; Cattell, 1984; Grimshaw and Mester,1988, among others) refers to a class of verbs which are supposed to be semantically bleached and lacking enough thematic force to function as predicates independently. For instance, English verbs "give" "take" and Chinese verbs "da" "chi" are all light verbs. When used together with different collocates, their literal meanings are bleached. Light verb constructions are common cross-linguistically thus show linguistic universality.

Just as in other types of languages, Light verbs are extensively used in Mandarin Chinese. Several linguists (Feng, 2005; Qiu, 2010; Wen, 2007; Zhang, 2013) have focused on Mandarin Chinese light verbs regarding their role and semantics. There are several prominent features attached to light verbs: in a syntactic sense, the light verbs reflect their syntactic functions with the aid of verb or verb phrase as the object as well as noun phrase as the object; in a semantic sense, light verbs exhibit vagueness of meaning or tend to be polysemous. The meaning focus is usually on objects and the meaning of the verb tends to be obscure and is usually decided by collocates followed.

The established studies on light verbs are almost defined in the generative linguistic sphere, emphasizing syntactic system, conception reconstruction and semantic bleaching (Chomsky, 1995). This paper attempts to put light verb study in a broader scope by exploring their meaning features with analysis of delexicalized features of some typical light verbs in Mandarin Chinese and adopt corpus-linguistic method to exhibit the delexicalization phenomenon.

\section{CHARACTERISTICS OF LIGHT VERB CONSTRUCTIONS AND SOME VOICES OF DELEXICALIZATION}

The meanings and functions of light verbs are both context-dependent. They are lexical items which provide simultaneously semantic contributions and discourse functions to their constructions, and are best accounted for against a theoretical background in which grammatical function is taken to be continuous with, rather than dichotomously opposed to, semantic content. A light verb construction (LVC) is headed by a verb which typically has a very wide distribution - it is polysemous and are usually associated with a wide variety of patterns of argument selection.

Jespersen and his followers hold that light verbs have no semantics of their own. This paper aims to challenge this point and argues that light verbs display lexical polysemy and show "constructional meaning" in LVCs. We cannot always isolate a distinct meaning of the light verb apart from the construction that it heads. In other words, it is only in construction with specific arguments that the contribution of the light verb can be determined. In this sense, as a result light verbs are delexicalized.

Sinclair launched the delexicalization conception system in 1991(Sinclair, 1991). Delexicalization is a phenomenon that high-frequently used words lose 
their literal semantic content. This phenomenon reflects meaning relationship among lexical collocations and represents co-selection of different forms in the communicative process. Delexicalization is the result of multiple meaning infections in a context. It is especially common for high frequently used words, including verbs, nouns, modifiers as well as intensifiers. Most light verbs are high frequently used.

\section{CASE STUDY}

\subsection{The meaning and delexicalization features of "da"}

According to the Contemporary Chinese Dictionary and the 800 words in Contemporary Chinese, there are 21 meanings when "da" acts as a verb. Each multi-words unit, or verb collocation has a unitary meaning:

1) Knock or hit by hand or using a tool: da gu (beat a drum)

2) Break, smash (container, egg, etc.): wan da le (The bowl is broken.) ji fei dan da (The hen has flown away and the eggs are broken.)

3) Beat, fight, attack: da jia (fight)

4) Deal with sb. or sth.: da guan si( go to court) da jiao dao( come into contact with)

5) Construct, build: dab a (construct a dam)

6) Make ( articles of daily use or food) : da jia ju (make furniture)

7) mix, stir: da xianer (stir filling)

8) tie up: da bao guo (pack one's luggage)

9) knit, weave: da mao yi (knit a sweater)

10) draw, paint, make a mark on: da la (wax) da wen hao (put a question mark)

11) Open, dig da jing (dig a well), da yaner (drill a hole)

12) Hoist, raise dad eng long (carry a lantern) da san (hold an umbrella)

13) Send, dispatch da dian hua (make a phone call)

14) Buy da jiu (buy a drink) d ache piao (buy a ticket)

15) Catch, hunt day u (catch a fish)

16) Reap, collect da chai (gather firewood)

17) Calculate, work out da cao gao (work out a draft) da zhu yi( think of a plan)

18) Do, engage in da zaer (do odds and ends)

19) play da qiu (play ball)

20) Go through some physical action da ha qian (yawn)

21) Adopt, use da bi yu (use a metaphor) da guan qiang (talk like a bureaucrat)

It seems that the meaning of "da" as a single word has become obscure. Only when it collocates with other words can the concrete meaning be displayed. The delexicalizing features of "da" are reflected more apparently through the analysis based on CCL Corpus.
I collected 500 concordances with 48926 Chinese characters from CCL. "da" is the node word. One concordance is selected every other 10 ones. The corpus - driven analysis shows that there are 4 types of usages with the delexicalizing features to varying degrees. The first type of usage of "da" has the basic meaning "Knock or hit by hand or using a tool", such as "ou da" "da gu" "quan da jiao ti". There are 18 such cases among 131. There isn't any delexicalizing feature shown in this type of usages. The second type usage such as "da zhe" "da zhang" "da pi lao zhan" "da yin" "da gong" "da zi" "da bao" "da kai" "da suan" "da guan si" "da sheng zhang" "da ying" "da bai" "da suan pan" "da jiao dao" "da ban" "da ge" "da tai ji quan" "da dian hua" "da zhao hu" "da ya mi" seems to be a unit of meaning. These collocations has been established units of meaning. As a light verb, "da" has even lost its basic meaning "hit,beat" whose meanings are decided by the collocates. In other words, it is the "embedded meaning" in the established collocations that makes "da" displays delexicalizing features. There are 61 such cases among 131. The third type usages are "da xia、 da hao" "da po" "da chu" . There are 31 such cases among 131. They are typical resultative construction usage in which "da" is used to yield results with the aid of some prepositions. Its delexicalizing features as a verb are also displayed in this type of usage but seem to be less typical compared with the fourth type. The fourth type usages are "da ban zhang qi dao mei yi ge zhan shi" "da ming tian qi" "da zhe wang dong zou". There are 4 cases among 131. "da" in these collocations is a colloquial use with the Northern China dialectic feature. The lexical features as a verb has almost disappeared and it has transferred into a preposition with the meaning of "since" or "from".

\subsection{The meaning and delexicalization features of "chi"}

According to the Contemporary Chinese Dictionary and the 800 words in Contemporary Chinese, there are 10 meanings when "chi" acts as a verb.

1) Put food into one's mouth, chew and swallow, eat: chi fan (have dinner)

2) Eat at a place that sells food; eat by certain standard: chi shi tang (have meals at a dining hall) chi xiao zao eat from a special pot

3) Live off, live on chi lao ben (live off past achievements)

4) Absorb liquid, soak up zhe zhong zhi bu chi mo (This paper does not absorb ink well)

5) One object entering into another chi dao (penetration of a cutting tool)

6) (Mostly in war or a chess game) annihilate, wipe out chi diao di ren yi ge tuan(wipe out an enemy regiment)

7) Understand, grasp ta de xin si wo hai chi bu 
tou(I'm not sure what's in his mind)

8) Endure, withstand, take chi de xiao (can endure or handle hardship)

9) Suffer, incur, bear chi kui (suffer losses) chi jing(be startled)

10)Consume, exhaust chi li ( laborious )chi jin (energy-consuming)

With the basic meaning of "put food into one's mouth, chew and swallow", the first type of usage of "chi" does not display any delexicalizing feature. There are 76 cases among 113 ones. The usages such as "chi cai zheng fan 、 chi zi zhu" mean "eat by certain standard". "cai zheng fan" or "zi zhu" are not the objects of the verb but a standard or a way of eating. People do not use "he (drink)zi zhu" just because "he zi zhu" is not a unit of meaning. So in the second type "chi" has some delexicalizing features as a single verb. As for the third type, "chi jing , chi liv chi ku、 chi bai zhang" seem to be more holistic and more established as collocations which are verb units. "chi" as a single verb in these multi-word verb units has nearly lost its original meaning. So this is a typical delexicalization. The fourth type is the resultative construction usage. "chi de xiao" or "chi tou" indicate a certain result. The meanings are established. Each word as a grammatical element is also habitually formed and can't be altered. In other words, both the form and meaning are holistic. Thus the delexicalizing features are obvious.

\subsection{Discussion}

The previous corpus-based study of features and tendencies of delexicalized verbs in Chinese shows that the heavy-duty verbs, to varying degrees, lost their original semantic content and displayed typical semantic and structural patternings, and that such delexicalized usages have become the norm. Delexicalized verbs in Mandarin Chinese like "chi" and "da" gradually lack an independent meaning while the grammatical function tends to be stronger. It is worth noting that they have obtained a greater capability in collocation with other words.

In lexical acquisition process, which includes both English as a foreign language acquisition and Chinese as a second language acquisition in this paper, light verb acquisition is a difficult part. Lack of idiomaticity is a common weakness of the nonnative speekers' speeches. Due to the delexicalizing features, delexicalization contributes to the nativelikeness of everyday use of target language.
Therefore, if the light verbs are taken in, stored and processed as a holistic unit together with the other collocates that co-occur highly frequently, and learners do not attach particular meaning to particular verb, delexicalized verbs are believed to entail a delexicalized idiomaticity that they are preferred by speakers in use.

\section{CONCLUSION}

As a unit of meaning, the light verb constructions display semantics of their own and play grammatical and textual functions simultaneously. If light verbs are treated separately as a single word, their meanings tend to be vague. But if the functional and holistic view is taken, the light verbs show both semantic and structural patternings. There is a tendency that light verbs are delexicalized and the semantic contents are embedded in the other collocates that frequently co-occur with them.

Being nativelikeness is the standard of foreign language acquisition. Analysis of delexicalizing features of light verbs can be applied to vocabulary acquisition and the intake of some highly-frequent used verb collocations is an ideal way of light verb comprehension, storage and extraction.

\section{REFERENCES}

[1] Cattell,R.1984. Syntax and Semantics: Composite Predicates in English. London: Academic Press.

[2] Grimshaw, J., Mester, A. 1988. Light verbs and thetamarking. Linguistic Inquiry 19,: 205-232.

[3] Jespersen,O. 1965. A Modern English Grammar on Historical Principle. London: George \& Unwin.

[4] Partington, Alan. 1993. Corpus evidence of language change: The case of intensifiers. In Mona Baker et al. (eds.), Text and technology: In honour of John Sinclair: 177-92. Amsterdam \& Philadelphia: John Benjamins.

[5] Feng, Shengli. 2005. Light Verb Movement in Modern and Classical Chinese. Linguistic Sciences. 4(1):3-16.

[6] Qiu,Wei. 2010. Study on English Chinese Light verb structure from cognitive perspective. Foreign language education. 3:32- 36.

[7] Wen, Binli. 2002. A minimalist program for linguistic theory. Beijing: Foreign Language Teaching and Research Press.

[8] Wen,Binli. 2007. Syntax Features of light verbs. Modern Foreign Languages, 2:112-120.

[9] Zhang, Zhiyi, 2013.Syntax and Semantic study of Light Verbs. Foreign Language education, 2:17-20. 